\title{
Decision Making in Online Social Networks Minitrack
}

\author{
Valeria Sadovykh \\ PwC and University of Auckland \\ valeria.a.sadovykh@sg.pwc.com
}

\author{
Gabrielle Peko \\ University of Auckland \\ g.peko@auckland.ac.nz
}

\author{
David Sundaram \\ University of Auckland \\ d.sundaram@auckland.ac.nz
}

Online Social Communities and Networks (OSN) have become widely popular as a source of data or reference for those seeking advice. With accelerated speed there are more and more websites tapping into the 'wisdom of crowds' as a source of information that influences our everyday decision-making. The internet has revolutionized the manner in which individuals obtain the information they need to make decisions.

Using OSN can accelerate or decelerate the DM process for both individuals and communities through the accessing of data from multiple sources. While ample independent research exists on OSN and $\mathrm{DM}$, there is a lack of research into how online technology affects the making of decisions that have an impact on our lives. How do we use OSN in our most important everyday decision-making? The synergy of these themes provides a unique research perspective from which to take a fresh look at both DM research and the actual process of DM as it is affected by the use of OSN.

The main purpose of this is to explore and extend, as well as challenge, existing knowledge of OSN and DM. We hope to understand and ascertain whether OSN can support and empower users in their decisionmaking process and particular phases (1) identify and conceptualize new phases (if any) in the decision-making process that is integral to OSN conversations (2) explore the structure and sequence of decisionmaking phases arising out of the use of OSN
(3) identify biases, strengths and weaknesses of the human psyche that could be attenuated and/or enhanced through appropriate design of OSN for decisionmaking and (4) seek practical guidelines for the design of OSN that support blended decision-making processes that leverages the wisdom of crowds.

This mini track continues to attract interesting papers. This year we had a diverse range of papers.

The first paper, Unique Challenges of Decision-Making Process on Crowdfunding Platforms-An Exploratory Study examines how funders' decision making process is affected by different types of uncertainty related to the project initiators. Unlike traditional e-commerce where consumers buy a finished product, in patronage based crowdfunding platforms, funders invest in and buy a product that is yet to be finished. This creates a unique uncertainty based on project initiators' competence. Their results show that uncertainty based on project initiators' competence and opportunism increase product performance uncertainty.

The second paper, Is Negative Feedback Better than No Feedback? The Impact of Social Dynamics on Reviewers' Review Decisions identifies that only a few studies examine online reviewer's decision making. This paper directly measures social influences on reviewers' review decisions as well as on consumers' voting decisions. Contrary to traditional cognitive evaluation theory, they found that both positive and 
negative feedback may positively motivate reviewers review behaviors.

The third paper, The Message Influences Me More Than Others: Social Media Metrics, Third-Person Perception, and Behavioral Intentions examines how the presence of social media metrics affect perceived media influence on self and others (Third-Person Perception - TPP) and subsequent behavioral intentions to combat an environmental risk. Their results suggest that the presence of social media metrics reverses TPP, social media metrics increase compliant behavioral intentions through mediation by TPP, and the need to belong moderates the effects of social media metrics on TPP.

The fourth paper, A Differentially Private Matching Scheme for Pairing Similar Users of Proximity Based Social Networking applications identifies that pervasiveness of smartphones, connecting with users through proximity based mobile social networks has become commonplace in today's culture. In this world of ever-increasing concern for privacy, users are wary of openly sharing personal information with strangers. In this paper, they propose a matching scheme that accurately pairs similar users while simultaneously preventing malicious users from attempting to infer information. 